\title{
EL TURISMO RURAL COMO FORMA DE DESARROLLO SOSTENIBLE. EL CASO DE ARAGON ${ }^{1}$
}

\author{
Blanca LOSCERTALES \\ Dpto. de Geografía y Ordenación del Territorio \\ Universidad de Zaragoza
}

\begin{abstract}
Resumen: El espacio rural se caracteriza en la actualidad por una gran complejidad y su mantenimiento es un objetivo prioritario. Para ello es necesario contar con una población mínima cuya principal misión es la de mantener dicho espacio. La pluriactividad constituye una de las vías para mejorar el nivel de vida de la población y las actividades turísticas son una importante fuente de pluriactividad en el medio rural de montaña. La oferta de alojamientos rurales, aunque minoritaria en el conjunto de la oferta, es una actividad creciente. El artículo hace referencia a los resultados de diferentes encuestas y entrevistas realizadas en el ámbito de montaña aragonés. Proporciona un medio para la caracterización del fenómeno y para conocer cómo perciben esta actividad los habitantes rurales. Los datos de la encuesta se contrastan con los proporcionados por otras fuentes.
\end{abstract}

Palabras clave: patrimonio, pluriactividad, turismo rural.

\begin{abstract}
One of the most significant characteristics of country life nowaday is its complexity. At the same time its survival has become a major priority. From this point of view outcomes the necessity for maintaining a minimun population on who could rely the responsability for maintaining this living style alive.The development of a wide variety of activities appears to be a good way for improving the living standars at these sites and any type of enterprise related with tourism becomes an important source for such activities, specially at the mountains countryside. The offer for rural loogings, even though it represents a minimum part of the touristical offer, has demonstrated an increasing trend. This article refers to the results of different polls and interviews which had place in the mountains of Aragon. It leads the path to characterise this phenomenum and to acknowledge how the people of the mountains feel about this sort of activities. The results of the inquiry are compared with others obtained from different sources.
\end{abstract}

Key Words: heritage, wide variety of activities, rural tourism.

1 Este artículo forma parte de la investigación realizada en el marco del Proyecto de la DGICYT (Ps. 94-0060 «Desarrollo sostenible y medio ambiente en las áreas de montaña. El turismo rural». 


\section{EL ESPACIO RURAL Y SU TRANSFORMACIÓN}

No es fácil llegar a una única y comprensiva definición que abarque la complejidad que caracteriza en la actualidad al espacio rural. Con frecuencia se le considera una magnitud económica residual que, desde 1970, se encuentra en plena crisis, aunque no siempre sucede así. La agricultura constituye como todo el conjunto del sector primario una actividad que está en decadencia y cabe también anotar que el modelo social se ha modificado profundamente. Por otra parte se incrementa la consideración de los aspectos positivos que pueden señalarse: un espacio cultural tradicional que cumple funciones importantes, ofrece un mundo menos contaminado y contribuye a forjar una diversidad cultural y una identidad nacional. El mantenimiento de los espacios rurales constituye un objetivo prioritario y para ello se considera imprescindible contar con una determinada población cuya principal misión es salvaguardar el patrimonio rural.

Los procesos recientes son en muchas ocasiones contradictorios, ya que, junto a la despoblación de unas áreas, es cada vez más fuerte la urbanización de otras; se produce de forma selectiva el renacer de determinados municipios - en muchas ocasiones vinculados al turismo- en los que se incrementa la residencia secundaria, o de aquellos otros situados próximos a determinadas áreas urbanas y metropolitanas que se benefician de los procesos de exurbanización y de descentralización de determinadas funciones. La despoblación afecta en particular al mundo rural profundo y en especial a las áreas de montaña no impactadas por la aparición de nuevas actividades (GARCÍA RAMÓN y ot., 1995). En ellas es patente el envejecimiento poblacional, la masculinización y la falta de reemplazamiento generacional. La problemática que afecta a gran parte del mundo rural, plantea la necesidad de una actuación conjunta de diferentes agentes económicos públicos y privados. La pluriactividad y el crecimiento del sector terciario constituyen una esperanza en los municipios y en los asentamientos en los que este sector se desarrolla - y ello es en ocasiones posible si se vincula a la actividad turística- previéndose que puedan convertirse en lugares dinámicos y de inmigración. El espacio rural ciertamente no puede ser siempre calificado como una magnitud económica residual.

\section{LA PLURIACTIVIDAD Y EL DESARROLLO DE LAS ÁREAS RURALES}

El desarrollo integrado de las áreas rurales es percibido como el modo de salir de la crisis generalizada. Para ello hay que diversificar las actividades económicas, pero también garantizar la calidad de los servicios - al menos de los básicos- a la población residente, especialmente de los servicios educativos, sanitarios o de ocio, como un factor encaminado a frenar la emigración. La mejora de la red de infraestructuras y transportes es también un factor importante para acabar con el aislamiento de muchas áreas. Estas actuaciones podrán contribuir al mantenimiento de una población rural que tiene como misión ser cuidadora y conservadora del espacio rural: «jardineros de este espacio». 
Una de las vías para mejorar el nivel de vida de la población rural es potenciar un modelo de desarrollo basado en la práctica de la pluriactividad y en el que sus principales actores e iniciativas surjan del propio medio local. La pluriactividad en el espacio rural no es reciente, pero se ha intensificado vinculándose a las mutaciones que han tenido lugar al emerger con fuerza un sistema productivo diversificado en el que ésta constituye un factor muy positivo que puede evitar la despoblación. El mundo rural «evoca todo un tejido económico y social con un conjunto de actividades de lo más diverso como agricultura, artesanía, pequeñas y medianas industrias, comercio y servicios» (COMISIÓN EUROPEA, 1988) y es previsible que en el futuro las actividades secundarias y terciarias sigan en ascenso en muchas áreas rurales y que la actividad agraria disminuya paralelamente su peso específico.

Desde esta perspectiva, el desarrollo de la actividad turística es y será un importante factor que puede contribuir al incremento del nivel de vida, al generar nuevas posibilidades de ingresos. El turismo y las diferentes actividades vinculadas a él, no siempre deben sustituir a las actividades agrícolas, sino darse de manera complementaria. La plurifuncionalidad que se predica como nota característica del espacio rural en la actualidad, debe permitir que sea compatible la utilización recreativa y la función productiva del territorio. En este contexto, el turismo en los espacios rurales, se ha convertido en un verdadero motor de desarrollo, aunque es clara la inexistencia de un acuerdo generalizado sobre el modelo adecuado de gestión.

Un desarrollo integral de carácter endógeno en el medio rural se basa en la explotación de los recursos del medio natural, de los recursos demográficos y de aquellos otros que se han ido forjando durante la historia y del resto de los recursos que puedan tener un valor económico. Su objetivo es conseguir un impacto positivo en la población autóctona y un equilibrio ambiental, social y económico desde la perspectiva de un desarrollo sostenible, ya que éste constituye en la actualidad una variable explicativa básica en cualquier modelo de desarrollo económico; y ello supone preservar los ecosistemas y todos aquellos valores no sólo de carácter natural sino también culturales o patrimoniales y recuperar y fomentar las tradiciones y todo lo que contribuya a destacar la personalidad de una determinada área. Por esta razón, conservación y desarrollo no pueden ser concebidos como conceptos antagónicos y su compatibilidad es esencial para dinamizar los recursos locales (TROITIÑO VINUESA, 1993). El patrimonio - natural y cultural-, no es solo un conjunto de bienes objeto de inventario, sino que puede ser explotado; y las actuaciones dirigidas a su conservación y recuperación tienen un impacto económico. El territorio constituye el principal recurso de la actividad turística, además de su soporte y escenario; en la práctica de un turismo de carácter cultural o ambiental, el paisaje rural constituye un importante factor. Es testimonio de los hombres que lo crearon y como bien insustituible hay que protegerlo de diferentes agresiones entre las que pueden mencionarse los cultivos no adecuados, las realizaciones con un fuerte impacto ambiental o las construcciones incontroladas. La actividad turística sólo contribuirá a la conservación del medio si se gestiona correctamente, tratando de evitar los conflictos que pudieran ocasionarse, ya que en caso contrario pueden producirse procesos de deterioro irreversibles. 


\section{EL TURISMO RURAL}

No es fácil identificar qué es el turismo rural, ya que constituye un concepto muy amplio que admite múltiples variables. No existe una definición universalmente aceptada que sea compartida por la comunidad científica ni por las administraciones públicas. En cada ámbito territorial existen estructuras muy variadas que colaboran a hacer la realidad cada vez más compleja, tanto en los que se refiere a la oferta como a la demanda. No hay un acuerdo generalizado entre los diferentes países, ni siquiera, muchas veces, dentro de un mismo país. En España existe, en las diferentes Comunidades Autónomas, una gran diversidad de leyes y normas o de denominaciones como en el caso de los alojamientos.

La expresión turismo rural es reciente y surgió para distinguir una alternativa de opción turística que responde al creciente interés de los ciudadanos por el patrimonio y la cultura rural; satisface la demanda de espacios abiertos para la práctica de gran número de actividades; constituye un factor muy positivo en la creación de empleo o para el incremento de la renta con un carácter de complementariedad económica. Puede identificarse como el uso planificado de los recursos de una área rural que favorece una mejora de la economía y del medio ambiente. Esta modalidad turística se opone al turismo de masas y de ahí el nombre de turismo alternativo. Constituye un modelo "postfordista» que aplica el principio de la sostenibilidad (VERA REBOLLO y ot., 1997). La Unión Europea, entiende que el concepto de turismo rural es más amplio que el de agroturismo o turismo en granjas, ya que afecta a todas las clases de turismo en el ámbito rural. Las sociedades receptoras de los turistas y ellos mismos se enriquecen mutuamente en muchos y en muy diferentes aspectos. El análisis de esta actividad se ha incorporado recogiendo una nueva realidad socioeconómica y espacial- en los estudios e investigaciones científicas sobre el turismo o el espacio rural.

La gestión turística es realizada por la población local y se dirige a una demanda que elige el espacio rural por muy diversos motivos. Este modelo turístico requiere la creación de equipamientos y servicios a pequeña escala ubicados de manera difusa en el territorio; es decir, huyendo de la centralización en uno o en unos pocos lugares. La oferta de alojamientos rurales es importante, pero hay que complementarla con otra de servicios de tipo social, cultural y deportivo que, junto con el alojamiento, integren el paquete turístico rural.

A la actividad turística en el medio rural se le aplican diferentes nombres: agroturismo, turismo verde, turismo ecológico, turismo de interior o de montaña, turismo activo, turismo deportivo o turismo ambiental. La variedad de denominaciones puede llevar a confusión, sobre todo si se considera que por turismo rural se entiende muchas veces una modalidad de alojamiento diferente de la oferta convencional, pero que se encuentra incluso como complemento de aquella en áreas próximas al litoral; y por otro lado el turismo de montaña incluye el turismo invernal o blanco, alejado en la actuali- 
dad de lo que es un modelo de turismo alternativo, aunque quizá no lo estuvo en su origen. Constituye una modalidad turística vinculada cada vez más a un modelo de turismo de masas que no es difuso y, especialmente en la modalidad de esquí alpino, no siempre es respetuoso con el medio natural. No es gestionado por agentes locales en la mayoría de los casos, aunque sí beneficia a la población local que actúa habitualmente como sujeto y no como agente económico. Es relevante el cambio que se ha producido en la política comunitaria desde la celebración en 1990 del año europeo del turismo que favoreció la aparición de diferentes propuestas especialmente orientadas a promover nuevos espacios y modalidades turísticas: turismo rural, verde, blando, cultural, de montaña, agroturismo, social, etcétera. Con este motivo nacieron diferentes proyectos de turismo en el medio rural y se inició un tratamiento exhaustivo del turismo rural en los textos de la Unión Europea.

El turismo en las áreas rurales europeas no es un hecho nuevo. En muchos países cuenta con una larga tradición; en otros, como España, al menos en su caracterización más moderna, constituye un fenómeno más reciente. En un período anterior era frecuente el retorno, en épocas concretas, de los antiguos emigrantes o de sus descendientes para visitar a sus familias o buscando sus propias raíces. A esta práctica cabe añadir alguna actividad específica orientada al ocio, como la utilización de balnearios - de los que queda constancia muy remota - el turismo cinegético, o incluso el hospedaje en una vivienda rural de la población urbana. La utilización generalizada del medio rural para el ocio se incrementó a partir de 1980, cuando en el mundo rural español se inició una nueva situación especialmente motivada por el ingreso en la Unión Europea, que ocasionó una disminución en la rentabilidad de muchas de las explotaciones agrarias y un peligro para su supervivencia. El desarrollo turístico obedece a causas internas y externas al propio medio rural entre las que cabe citar la crisis del turismo tradicional, la aparición de una conciencia medioambiental y que es una actividad económica cuyo crecimiento se vincula al declinar de la actividad agraria, y a que ésta no ha sido capaz de solucionar los problemas económicos y sociales de las áreas rurales deprimidas. La utilización turística del espacio rural se convierte, en este contexto, en una alternativa económica.

El turismo rural cobra importancia y constituye una importante fuente de pluriactividad que genera además gran número de actividades complementarias, por lo se erige en un sector económico de carácter motriz en el conjunto de la actividad económica y constituye en mayor o menor medida un verdadero motor de desarrollo. Las actividades impulsadas son muy variadas; se asocian generalmente a la complementariedad económica, pero incluso están vinculadas a un proceso de sustitución de rentas. Cabe reseñar entre ellas las diferentes formas de alojamiento, restauración, actividades deportivas u otras actividades de ocio y una amplia gama de actividades artesanas que favorecen la recuperación de las raíces culturales. La actividad turística, favorece la práctica de una agricultura menos intensiva y que utiliza medios de producción más naturales. Los productos conseguidos son requeridos por los turistas que acuden a estas áreas y se convierten en una fuente de ingresos mediante la comercialización directa por los agricultores o su utilización en la actividad turística. Otro de los importantes impactos que se derivan de la 
práctica turística es que incorpora como población activa a una mano de obra - jóvenes, mujeres, ancianos incluso- que de no existir la actividad turística no se produciría. Se da también la redefinición de las relaciones sociales y laborales en el seno de la explotación agraria familiar, modificando especialmente el papel otorgado a la mujer.

\section{TURISMO RURAL Y TERRITORIO}

En cualquier actividad de carácter económico se produce una íntima relación con el territorio que le sirve de soporte; la calidad del mismo y su dotación desde un punto de vista físico y humano, es un factor muy importante para la aparición de nuevas actividades, pero cuando dichas actividades se desarrollan, pueden producir un fuerte impacto territorial de carácter espacial y socioeconómico. En el turismo rural se da ciertamente una relación íntima entre el turismo, el territorio y la sociedad (MARCHENA GÓMEZ, 1987). La nueva utilización del espacio rural favorece la superación de resistencias y conflictos entre los diferentes sujetos que utilizan este medio o entre las diferentes actividades que lo tienen como soporte.

La práctica turística tiene consecuencias directas en la definición y catalogación de los recursos de un lugar; los habitantes rurales adquieren conciencia de su propio patrimonio, especialmente al darse cuenta de que personas de fuera lo valoran y este hecho constituye un factor muy positivo para su conservación; la actividad turística favorece que se lleven a cabo actuaciones para la renovación y recuperación del hábitat rural, tanto en las viviendas destinadas a alojamientos de turismo rural, como en las residencias secundarias y en muchas de las viviendas de carácter primario. La mejora del patrimonio es a su vez factor que garantiza la continuidad de la actividad turística. Por ello, la necesidad de protección del territorio es una constante en los estudios sobre turismo en el espacio rural.

La formación de recursos humanos constituye también un importante factor para el buen funcionamiento de cualquier actividad de carácter económico. Uno de los objetivos del año europeo del turismo comunitario, fue conseguir un mayor grado de profesionalidad en la gestión del turismo rural y a ello se destinaron importantes recursos La formación de la mujer, merece una atención especial; se incorpora a las nuevas actividades y es considerada como un agente importante de la conservación del medio nat. ural y del medio ambiente.

\section{NOTAS SOBRE EL TURISMO RURAL EN EL ÁMBITO DE MONTAÑA ARAGONÉc}

El turismo en el espacio rural ha experimentado un fuerte auge en las áreas de mon taña aragonesas, generalmente en la línea de un turismo sostenible. Las asociaciones df 
titulares de vivienda rural ${ }^{2}$, el número de asociados y el número de empresas dedicadas a la gestión o explotación del espacio rural han tenido paralelamente un fuerte incremento; las empresas dedicadas a la práctica de actividades deportivas han aparecido especialmente en el ámbito del Pirineo y del Sistema Ibérico; son fruto de la iniciativa de jóvenes, amantes del deporte, originarios del lugar. La actividad turística ha servido para arraigarlos en el territorio, al proporcionarles un empleo, muchas veces de carácter estacional, ya que se requieren unas determinadas condiciones atmosféricas que están vinculadas al buen tiempo, lo que las convierte en muchas ocasiones en un complemento estacional del esquí. La utilización del medio rural como espacio de consumo ha obligado a su protección mediante la normativa pertinente que se ha intensificado en los últimos años ${ }^{3}$.

El turismo rural persigue unos objetivos prioritarios de carácter social, económico y ambiental y se plantea también captar diferentes segmentos de una potencial demanda a la que deben orientarse los productos turísticos. La investigación realizada en las áreas de montaña de Aragón refrenda la progresiva consecución de los objetivos enumerados aunque crece también y, a medida que se desarrolla y perfecciona la actividad, la crítica a algunos aspectos.

\section{Caracterización de la oferta de alojamiento}

El análisis efectuado se ha centrado en las áreas de montaña del Pirineo y del Sistema Ibérico. Son áreas que han sufrido fuertes pérdidas demográficas durante este siglo y que han sido recientemente impactadas en mayor o menor medida por el desarrollo de diferentes actividades turísticas (figura 1$)^{4}$. La oferta de alojamiento es muy variada y se ha incrementado de manera paralela a la ocupación turística del territorio (tabla 1). Las viviendas de turismo rural tienen una representación importante en el Sobrarbe y en la Ribagorza, pero han crecido también en otras comarcas como Gudar-Javalambre, Albarracín, Maestrazgo, Matarraña, o en otras áreas de la región aragonesa como el Somontano de Barbastro. Los alojamientos rurales se ubican en muchas ocasiones en edi-

\footnotetext{
2 En marzo de 1996 se celcbraron en Daroca las Primeras Jornadas de Turismo Rural en Aragón, considerando esta actividad como un necesario complemento para el mundo rural. Las aportaciones de estas Jornadas fueron inestimables para el conocimiento de la situación, problemas y expectativas del turismo rural. La conferencia inaugural fue pronunciada por el Doctor BIELZA DE ORY, investigador de este proyecto.

3 No hay posibilidad de realizar una enumeración pormenorizada en este artículo. Cabe afirmar que la normativa trata de regular el comportamiento de los diferentes agentes turísticos y de la demanda y muy especialmente de contribuir a la conservación del territorio, evitando los problemas que pudieran derivarse de una gestión inadecuada. La legislación aragonesa en materia de turismo y medio ambiente que ha ido apareciendo en los últimos años es un fiel reflejo del interés que despiertan estas cuestiones y de la necesidad de su regulación ante una nueva realidad social y espacial como es el incremento de las más diversas modalidades turísticas en el espacio rural. En cl Boletín Oficial de Aragón de los últimos años puede comprobarse el hecho afirmado.

+ La figura 1 muestra la evolución demográfica en las comarcas aragonesas que tienen toda o una parte de su territorio de montaña; por ello, aparecen comarcas completas y otras que sólo están representadas parcialmente. La tabla 1 y la figura 1. han sido realizados por Isabel Bea Hortal.
} 


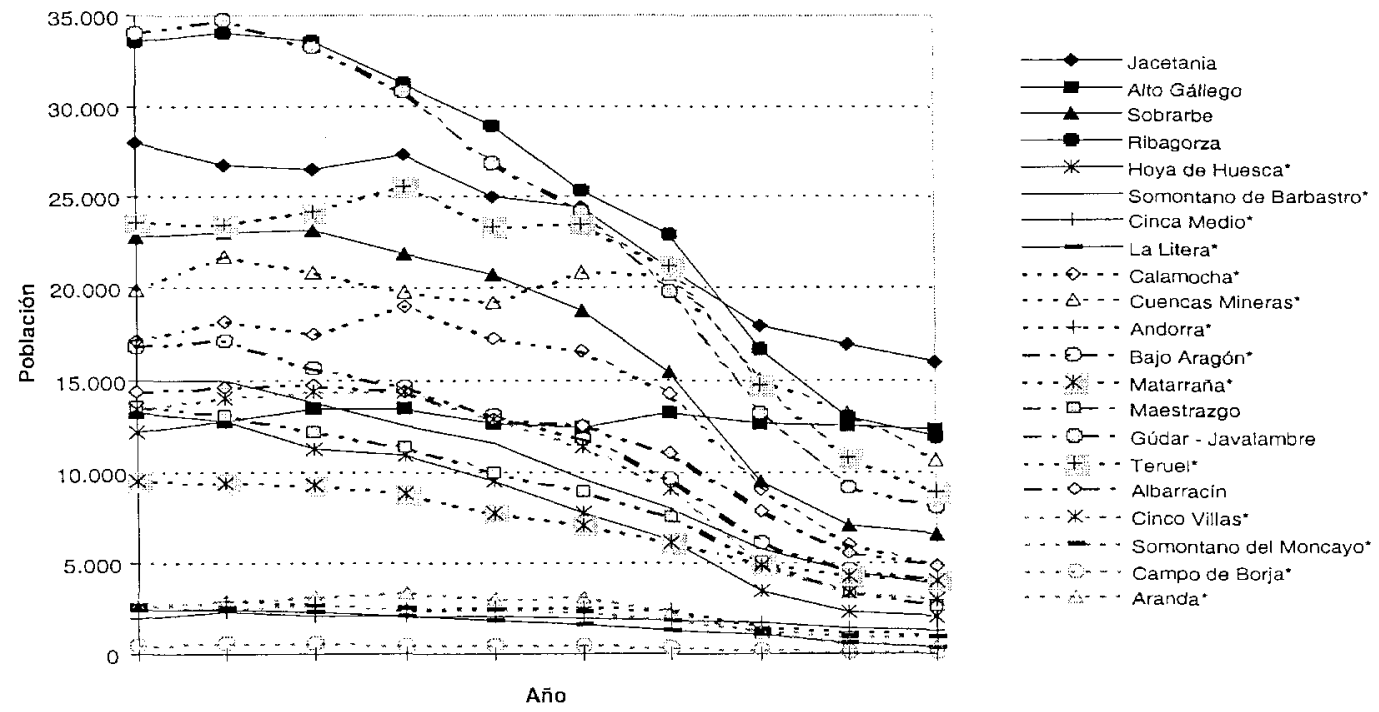

* No se incluye la comarca completa sino el área de montaña existente en la misma Fuente: Censos de población 1900-1991. I.A.E./D.G.A. Elaboración propia

Figura 1. Evolución demográfica áreas de montaña

Tabla 1. N. Total de plazas turísticas, según tipo de alojamiento,en las áreas de montãna aragonesas

\begin{tabular}{|c|c|c|c|c|c|c|c|}
\hline COMARCA & $\begin{array}{c}\text { ESTABLECIMIENTOS } \\
\text { HOTELEROS }\end{array}$ & $\begin{array}{c}\text { APARTAMENTOS } \\
\text { TURISTICOS }\end{array}$ & CAMPING & ALBERGUES & V.T.R. & $\begin{array}{l}\text { RESIDENCIA } \\
\text { SECUNOARIA }\end{array}$ & TOTAL \\
\hline Jacetania & 2.848 & 938 & 4.552 & 320 & 106 & 48.310 & 57.074 \\
\hline Alto Gállego & 1.748 & 79 & 1.874 & 242 & 41 & 16.095 & 20.079 \\
\hline Sobrarbe & 2.598 & 0 & 7.365 & 573 & 1.108 & 5.605 & 17.350 \\
\hline Ribagorza & 2.694 & 353 & 3.164 & 299 & 1.060 & 17.285 & 24.855 \\
\hline Hoya de Huesca* & 188 & 0 & 0 & 0 & 25 & 6.270 & 6.483 \\
\hline Somontano de Barbastro* & 206 & 0 & 804 & 192 & 165 & 3.455 & 4.822 \\
\hline Cinca Medio* & 0 & 0 & 0 & 0 & 22 & 1.040 & 1.062 \\
\hline La Litera" & 0 & 0 & 0 & 0 & 0 & 1.150 & 1.150 \\
\hline Calamocha* & 0 & 0 & 0 & 0 & 28 & 7.665 & 7.693 \\
\hline Cuencas Mineras* & 202 & 0 & 90 & 0 & 49 & 12.960 & 13.301 \\
\hline Andorra* & 0 & 0 & 0 & 0 & 0 & 1.830 & 1.830 \\
\hline Bajo Aragón & 206 & 0 & 340 & 0 & 62 & 7.780 & 8.388 \\
\hline Matarraña" & 172 & 0 & 0 & 0 & 139 & 5.005 & 5.316 \\
\hline Maestrazgo* & 280 & 0 & 0 & 0 & 129 & 5.980 & 6.389 \\
\hline Gúdar - Javalambre & 1.108 & 0 & 534 & 83 & 508 & 24.530 & 26.763 \\
\hline Teruel* & 108 & 0 & 0 & 0 & 104 & 10.925 & 11.137 \\
\hline Albarracin & 686 & 0 & 440 & 0 & 248 & 12.500 & 14.274 \\
\hline Cinco Villas" & 166 & 0 & 0 & 0 & 91 & 4.575 & 4832 \\
\hline Somontano del Moncayo* & 44 & 0 & 0 & 0 & 0 & 1.855 & 1.899 \\
\hline Campo de Borja* & 0 & 0 & 0 & 0 & 0 & 460 & 460 \\
\hline Aranda* & o & 0 & 0 & 0 & 6 & 1.700 & 1.705 \\
\hline TOTAL & $13 . \overline{354}$ & 1.370 & 19.154 & 1.709 & 3.891 & 197.375 & 236.863 \\
\hline
\end{tabular}

Fuente: - Censo de Viviendas de Aragón 1.991, ed. I.A.E. / D.G.A., Zaragoza, 1.996.

- Guía de Servicios Turísticos de Aragón 1.999, ed. Departamento de Economía y Fomento de la D.G.A., Zaragoza, 1.998.

- Elaboración Propia 
ficios de más de cien años (figura 2); así sucede en el Alto Aragón, área en la que el turismo rural ha tenido una gran significación para la recuperación y posterior refuncionalización de una parte del patrimonio arquitectónico, permitiendo su explotación económica y confirmando las sinergias positivas entre turismo y patrimonio. Las viejas casas del Pirineo destacan por su solera, en muchas de sus fachadas lucen escudos de armas, testigos del abolengo de sus antiguos moradores. Hoy sus descendientes luchan por la conservación de un patrimonio de indudable valor arquitectónico y paisajístico. El turismo rural es en este contexto una actividad nada despreciable de cara a la mejora y permanencia de un paisaje rural, muchas veces en crisis.

La oferta de plazas en alojamientos hoteleros alcanza sus máximos valores en las comarcas de la montaña pirenaica de la Jacetania, Alto Gállego, el Sobrarbe y la Ribagorza. La comarca de Gudar -Javalambre con 1.108 plazas- refleja el incremento de la actividad turística invernal. Las plazas de los campamentos turísticos también se han incrementado notablemente, tratando de responder a las características de un sector de la demanda; la oferta se concentra sobre todo en el ámbito pirenaico y se caracteriza por su estacionalidad. La residencia secundaria es sin duda la principal modalidad de alojamiento en el espacio de montaña aragonés; en muchas ocasiones se trata de las viviendas ubicadas en muchos antiguos edificios de utilización primaria, que quedaron vacíos por el éxodo rural o por la muerte de sus antiguos moradores y que en la actualidad han sido recuperados para la utilización temporal, ya sea por los emigrantes o sus descendientes o por personas ajenas al medio rural. Las urbanizaciones y nuevas construcciones destinadas a la utilización secundaria han aparecido con mayor intensidad en los asentamientos próximos a las pistas de esquí.

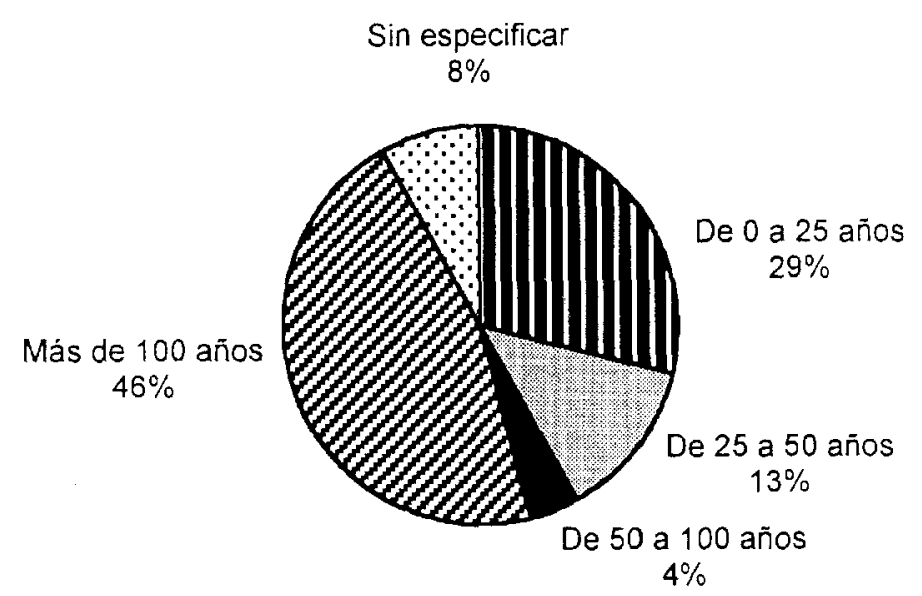

Fuente: Encuestas realizadas

Elaboración Propia

Figura 2. Antigüedad de los edificios 


\section{Dinámica turística en los alojamientos rurales ${ }^{5}$}

Esta modalidad de oferta de alojamiento ha llegado en algunos ámbitos a identificarse con el turismo rural. Reconociendo al fenómeno turístico en el espacio rural una perspectiva más amplia, no puede olvidarse su interés desde una óptica económica, ya que sirve como complemento de las rentas producidas por la actividad agraria o por otras actividades en el marco de la pluriactividad. Para identificar la situación se han realizado numerosas entrevistas y encuestas en diferentes lugares ${ }^{6}$ y de ellas pueden deducirse diferentes cuestiones. Los resultados obtenidos de la aplicación de la encuesta se han completado en el ámbito pirenaico con los resultados obtenidos de la elaboración de diferentes datos recogidos en la base de datos de la Central de Reservas de Turismo Verde.

De las encuestas y entrevistas realizadas se deduce que la motivación económica, es el principal factor, pero no el único que ha originado la puesta en marcha de esta actividad; la recuperación de un patrimonio arquitectónico en mal estado, el conocimiento de los resultados de esta práctica en otros lugares, el deseo de conocer y tratar a otras personas, o de participar en un movimiento asociativo y la oportunidad que ofrece a la mujer para ejercer un trabajo remunerado son también importantes factores (Tabla 2).

Las encuestas y entrevistas realizadas permiten aventurar diferentes conclusiones sobre las características de la población. Una primera afirmación es la importancia que tienen los agentes locales en la gestión de esta parcela de la actividad turística. Las personas del lugar representan un $54 \%$ del total de la población encuestada. Los titulares de vivienda rural son en muchas ocasiones personas con una edad media elevada; un 54,16\% de los encuestados tiene más de 50 años e incluso algunos de los mismos están jubilados (Tabla 3). Aunque esta situación ha despertado las críticas de los agentes turísticos de la oferta convencional, no puede olvidarse - y así lo han manifestado muchos de los entrevistados - que esta actividad ha favorecido que personas con muy débiles rentas - muchas veces de elevada edadpudieran conseguir unos ingresos complementarios; de aquí la función social que ha estado en la base de su desarrollo. Los titulares de mayor edad son fundamentalmente varones.

5 El Decreto 69/1997, de 27 de mayo del Gobierno de Aragón, aprobó el Reglamento sobre ordenación y regulación de Ios alojamienfos turísticos denominados Viviendas de Turisno Rural. Sustituye a una normativa anterior de las Viviendas de Turismo Rural (VTR) de 1986 que se realizó buscando diferentes objetivos como romper los desequilibrios territoriales, generar unas rentas complementarias a las proporcionadas por el sector primario, incrementar el empleo y recuperar el patrimonio arquitectónico. La nueva normativa se plantea espccialmente regular el proceso de autorización, apertura y control a que deben someterse todas las vivicndas de turismo rural y establecer unas características que deben respetar los alojamientos y definir los requisitos del prestatario del servicio.

6 Se han realizado numerosas encuestas, pero no todas han quedado recogidas en este artículo encaminado especialmente a conocer la situación de un sector especifico. La encuesta a la que hacemos referencia tuvo como objetivo principal identificar la dinámica turística en los alojamientos rurales y su impacto; se envió a los titulares de alojamientos de las diferentes Asociaciones. Las entrevistas a las que se hace mención han sido muy variadas. Los resultados obtenidos aparecen tanto incorporados al texto como en las tablas o en las figuras que se adjuntan. La limitación de espacio no permite representar en tablas o gráficas todos los datos. En el diseño de la encuesta y en la realización de algunas de las mismas colaboró Antonio Lacosta Aragüiés. 
Tabla 2. Motivación

\begin{tabular}{|l|c|}
\hline Completar sus ingresos anuales & $26,53 \%$ \\
Conocimiento de la práctica en otros lugares & $12,24 \%$ \\
Conocer gente & $12,24 \%$ \\
Recuperar un patrimonio en mal estado & $10,20 \%$ \\
Participar en un movimiento asociativo que promociona su comarca & $10,20 \%$ \\
Buscar salida a sus productos agricolas o artesanos mediante la promoción directa & $8,16 \%$ \\
Complementar la oferta turística & $4,08 \%$ \\
Aprovechar las ayudas institucionales & $4.08 \%$ \\
Oportunidad de trabajo para la mujer & $2,04 \%$ \\
Aprovechar la casa & $2,04 \%$ \\
\hline
\end{tabular}

Fuente: Encuestas realizadas

Elaboración Propia

Tabla 3. Edad y estudios realizados

\begin{tabular}{|c|c|c|c|c|c|c|}
\hline \multicolumn{2}{|c|}{ EDAD } & \multicolumn{5}{c|}{ ESTUDIOS REALIZADOS } \\
\hline GRLPOS & TOTALES & SIN ESTUDIOS & PRIMARIOS & MEDIOS & SUPERIORES & SIN ESPECIFICAR \\
\hline$<30$ & $4,16 \%$ & & $4,16 \%$ & $4,16 \%$ & & \\
$30-50$ & $41,66 \%$ & & $33,33 \%$ & $4,16 \%$ & & $4,15 \%$ \\
$>50$ & $54,16 \%$ & $4,16 \%$ & $33,33 \%$ & $4,16 \%$ & $4,16 \%$ & $4,16 \%$ \\
\hline
\end{tabular}

Fuente: Encuestas realizadas

Elaboración Propia

Los encuestados, como cabe esperar en una área rural y teniendo en cuenta la edad avanzada de una parte importante de los mismos, tienen un bajo nivel de estudios (tabla 3). Sin embargo hemos podido comprobar que se incrementa la formación para la práctica turística. Un $70 \%$ de los encuestados afirman haber seguido cursos específicos sobre turismo. Este hecho refleja el interés que despiertan estas cuestiones y también el dinamismo e inquietud que se trasmite desde las Asociaciones. Entre los cursos recibidos destacan los de elaboración de productos artesanos; es una confirmación de la conexión que se establece entre el turismo y la artesanía y de cómo el turismo promociona actividades que permiten el autoempleo y la pluriactividad. Cabe mencionar también los cursos de gestión económica y de atención a la clientela orientados a la mejora de los servicios prestados. Muchos de los cursos se han beneficiado de diferentes ayudas (Figura 3).

Del análisis de la composición profesional de los titulares de vivienda rural se deduce el elevado porcentaje de las amas de casa - un $25 \%$ del total de los encuestados-. La mujer valora muy positivamente la actividad turística ya que, además de integrarla en la actividad económica, le permite relacionarse con otras personas. La participación femenina es en realidad mucho más importante si se tiene en cuenta que son muchos los titulares varones que han declarado que son sus cónyuges las que se ocupan de la actividad especialmente en aquellas cuestiones que constituyen la prolongación de su habitual trabajo del hogar. Un 37,5\% de los encuestados se declaran trabajadores autónomos y solo un $16,6 \%$ agricultores. Los jubilados representan un $8 \%$ del total (Tabla 4 ). 


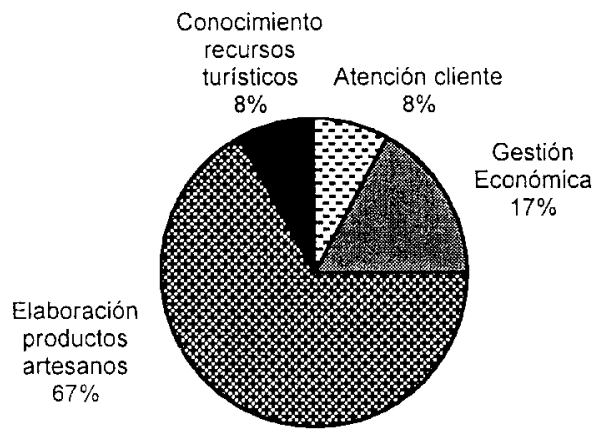

Fuente: Encuestas realizadas

Elaboración Propia

Figura 3. Cursos de formación específica realizados

Tabla 4. Profesión del titular del alojamiento rural

\begin{tabular}{|l|c|}
\hline PROFESION \\
\hline & \\
TRAB. AUTONOMO & $37,51 \%$ \\
AMA DE CASA & $25 \%$ \\
AGRICULTOR & $16,66 \%$ \\
JUBILADO & $8,33 \%$ \\
FUNCIONARIO & $8,33 \%$ \\
SINESPECIFICAR & $4,16 \%$ \\
\hline
\end{tabular}

Fuente: Encuestas realizadas

Elaboración Propia

La citada encuesta permite aproximarnos a otros hechos como el número de hijos por familia, con unos valores muy bajos, ya que un $66,6 \%$ de los encuestados tienen uno o dos hijos. Las respuestas obtenidas permiten valorar en poco la ayuda prestada por los hijos. Solo un 16,6\% afirman que sus hijos colaboran también. Las razones de esta baja representación pueden estar en que los hijos ya se han independizado de la economía familiar, dada la elevada edad de la población, o a que, respuesta muy repetida, son estudiantes de uno u otro nivel.

La mayor parte de los edificios y de las viviendas han debido ser remodelados para su orientación turística, hecho que ha tenido lugar especialmente a partir de los años ochenta (un $16 \%$ ) pero se ha incrementado notablemente en el decenio actual (un $54 \%$ ) reflejando los cambios acaecidos en el espacio rural y el interés por las nuevas modalidades turísticas (ver Figura 4). Un $42 \%$ de los encuestados afirman que han recibido subvenciones o créditos para ello. Las ayudas recibidas para la rehabilitación de los edificios son una muestra más de la positiva actuación del turismo sobre el patrimonio, en esta ocasión arquitectónico. No es fácil deducir lo que ha supuesto la ayuda sobre el conjunto de las 


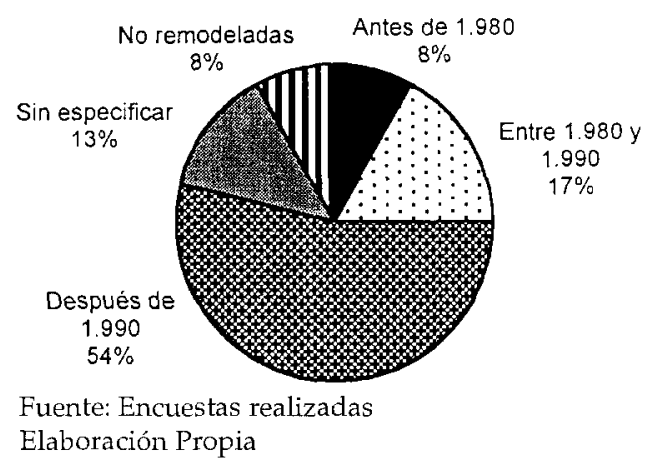

Figura 4. Fecha remodelación de los edificios

obras. Un $63 \%$ de los encuestados no aporta datos y, según se deduce de las restantes respuestas, los valores no suelen superar al $15 \%$ del conjunto de la obra. Pero no cabe duda de que la concesión de ayudas ha sido un importante incentivo para la realización de las mejoras, sobre todo teniendo en cuenta la favorable perspectiva económica posterior derivada de su rentabilización. Un $50 \%$ de los encuestados afirman la importancia de las ayudas concedidas por la Administración Autonómica. Los trabajos realizados en los edificios han contado esencialmente con una población activa local como queda reflejado en la totalidad de las respuestas. Ello confirma la conexión del turismo con otros sectores de la actividad económica y su efecto motriz sobre ellos.

Los titulares de alojamientos rurales encuestados perciben la actividad positivamente; destacan que pueden llegar a establecer una relación de amistad con sus clientes, ya que muchas veces repiten su estancia. Un $70 \%$ de los encuestados afirman tener una clientela de nivel medio y un $20 \%$ destaca el elevado nivel socioeconómico y cultural de la misma'. La Comunidad Valenciana constituye el área emisora fundamental de la clientela en algunas áreas como el Maestrazgo o Gudar-Javalambre. Las deficientes infraestructuras de transporte y comunicaciones y no sólo la distancia real de otras áreas de Aragón, son las responsables de este hecho. En el ámbito pirenaico las principales fuentes emisoras son Aragón, Cataluña y Madrid. Esta última valoración proporcionada por la encuesta es acorde con la que hemos obtenido tras la elaboración de los datos recogidos en la base de datos de la Central de Reservas de Turismo Verde. Según esta última fuente en 1997, el 96 $\%$ de la reservas en alojamientos rurales habían sido realizadas por población española y un 3,8 \% por europeos procedentes de los países de Unión Europea, casi en su mayoría franceses. Por Comunidades Autónomas destaca Aragón que proporciona un $39 \%$ del total de las reservas, Cataluña, un $19 \%$, Madrid un $13 \%$ y la Comunidad Valenciana un $11 \%$, a cierta distancia cabe citar Euskadi que proporciona un $3 \%$ y Navarra un $2 \%$. (Figura 5).

Los titulares encuestados enumeran también algunos aspectos negativos como el deterioro del material, la excesiva dedicación que requiere, la polémica con la hostelería tradicional y la estacionalidad en la dinámica de la utilización de las viviendas rurales. El 


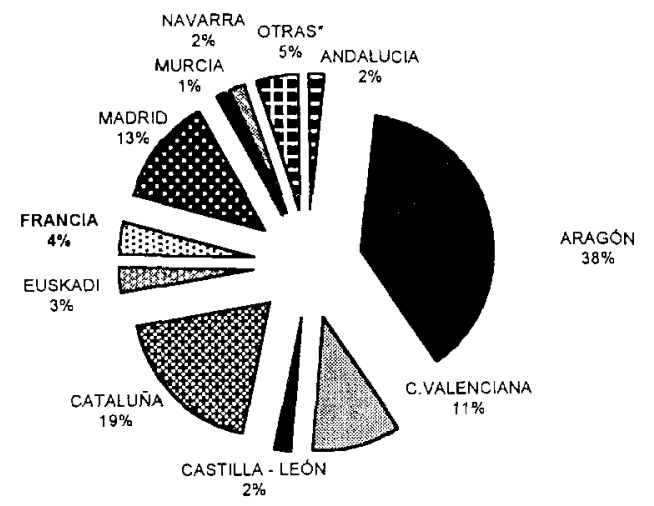

* Dentro del sector otras se reúnen aquellos lugares de procedencia que no superan el $1 \%$

Fuente: «Central de Turismo Verde» D.P.H., 997

Elaboración Propia

Figura 5. Origen de la clientela

verano es la estación más importante, pero también la demanda es importante durante la Semana Santa, el Fin de Año, el puente de la Constitución. Refleja la incidencia de los períodos vacacionales más cortos para equilibrar en cierta medida la ocupación durante el resto del año (Figura 6). La duración de la estancia no supera en el $89 \%$ de los casos la semana (Figura 7).

Las entrevistas mantenidas confirman también la generalizada y creciente preocupación de los diferentes actores turísticos por ofrecer una imagen de calidad del territorio mediante una adecuada publicidad; un $87,5 \%$ de los encuestados afirman que realizan publicidad a través de su asociación. Centran su actuación en atraer a los diferentes segmentos de la demanda, especialmente de los que permiten la consecución de sus objetivos, prioritariamente de carácter económico. Pero la idiosincrasia del fenómeno requiere que

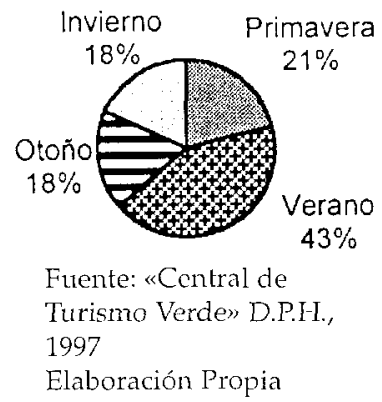

Figura 6. Estacionalidad 


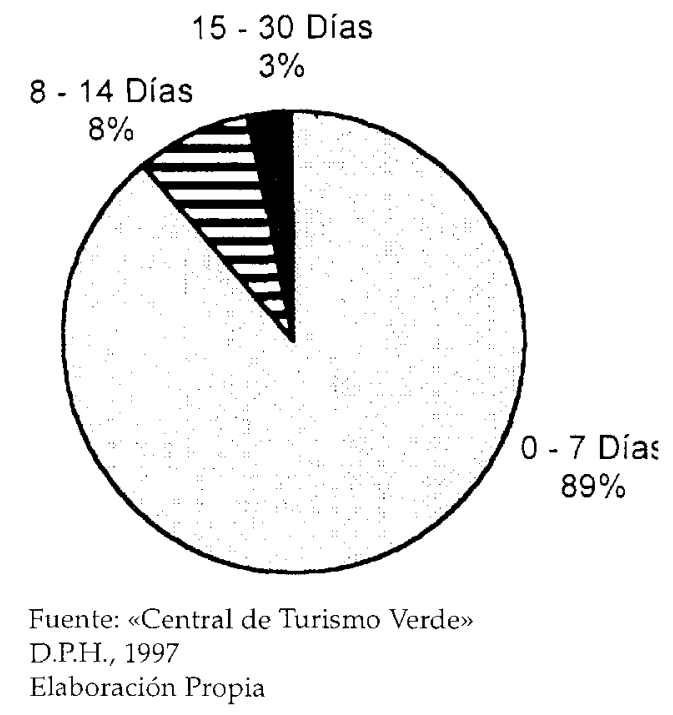

Figura 7. Duración de la estancia

sea contemplado en el marco del desarrollo sostenible y ello debe marcar las características y el número de la clientela.

A modo de conclusión general cabe afirmar que el turismo rural o en el espacio rural, constituye en España un fenómeno heterogéneo condicionado por diferentes factores. Las diferencias en calidad y promoción se deben a que han sido las diferentes Administraciones Autonómicas las que lo han impulsado y regulado sus modalidades de alojamiento (CANOVES VALIENTE y ot., 1995) La definición de unidades espaciales de planificación y de ordenación constituye un objetivo esencial (BOTE, 1992).

\section{BIBLIOGRAFIA}

AZQUETA OYARZUN, D. y PÉREZ Y PÉREZ, D. (Coords.) (1996): Gestión de espacios naturales. La deminda de servicios recreativos. Madrid.

BARDON FERNÁNDEZ, E. (1990): “Consideraciones sobre el turismo rural en España y medidas de desarrollo", Estudios Tutristicos, 130, 65-123, Madrid

BOTE, V. (1992): «Por una estrategia artesanal del turismo en el espacio rural» en El turismo rutral en el desarrollo local, 125-132, Madrid. 
CANOVES VALIENTE, G. y GARCÍA RAMÓN, M. D (1995): «Mujeres y turismo rural en Cataluña y Galicia ¿La nueva panacea de la agricultura?, El Campo, 133, 221-238, Bilbao.

COMISIÓN DE LAS COMUNIDADES EUROPEAS (1988): El futuro del mundo rurnl. Comunicación de la Comisión transmitida al Consejo y al Parlamento Europeo. 29 de julio de 1988 (COM (88) 501 final).

COMISIÓN DE LAS COMUNIDADES EUROPEAS (1991): Plan de medidas comunitarias a favor del turismo. COM (91) 97 final. Bruselas 23 de mayo de 1991.

DÍAZ PÉREZ FLORA, M. y FERNÁNDEZ FERNÁNDEZ, C. (1995): «Un análisis crítico de los requerimientos formativos en la implantación de actividades de turismo rural», Papers de turisme, 19, 91-100, Valencia.

FERNÁNDEZ MANCHÓN, F. (1995): «Agricultura y medioambiente: reflexiones desde el medio rural», El Campo, 133, 39-48, Bilbao.

GARCIA RAMÓN, M.D., TUYA PUJOL, A. y VALDOVINOS PERDICES, N. (1995): Geografía Rural. Madrid.

JURDAO ARRONES, F. (Coord.) (1992): Los Mitos del Turismo. Madrid.

KELLER, P. (1991): «Turismo rural ¿esperanza o ilusión? Una aportación vista desde la perspectiva de Suiza». Estudios turísticos, 110, 27-39, Madrid.

MONTEMAGNO y ARANCIO, (1991): «Turismo rural y agroturismo, el caso italiano», Revista de Estudios Turísticos, 110, 5-17, Madrid.

MARCHENA GÓMEZ, M. (1987): Territorio y turismo en Andalucia. Análisis a diferentes escalas espaciales. Sevilla

MARTiN GIL, F. (1994): «Nuevas formas de turismo en los espacios rurales españoles» en Estudios turisticos, 15-39, Madrid.

RAMiREZ GRAY, A. (1995): «La educación de la mujer rural como factor de desarrollo social» el $E l$ Campo, 91-106, Bilbao.

REYNA, S. et al. (1992): El turismo rural en el desarrollo local. Ministerio de Agricultura, Pesca y Alimentación. Madrid.

SAURI PUJOL, D (1995): «Recursos ambientales, pluriactividad y género en la reestructuración de los espacios rurales de los paises europeos", El Campo, 133, 25-38, Bilbao.

VALCÁRCEL-RESALT, G., TROITIÑO VINUESA, M. A. y ESTEBAN CAVA, L. (1993): Desarrollo rural, turismo y medio ambiente. Cuenca.

VERA REBOLLO (Coord.) et al. (1997): Análisis territorial del turismo. Madrid.

VILLARINO PEREZ, M. (1995): «Las mujeres rurales como agentes de la pluriactividad», El Camipo, 133, 11-24, Bilbao. 